\title{
Variability of Breast Density Assessment and the Need for Additional Imaging: A Comparison between Computed Mammography and Digital Mammography
}

\author{
Shaista Afzal Saeed, Imrana Masroor, Hina Iqbal, Saira Naz Sufian and Muhammad Awais \\ Department of Radiology, The Aga Khan University Hospital, Karachi, Pakistan
}

\begin{abstract}
Objective: To determine the variability of breast density assessment and the need for additional imaging using computed radiography (CR) mammography versus digital radiography (DR) mammography.

Study Design: Cohort study.

Place and Duration of Study: Department of Radiology, The Aga Khan University Hospital, Karachi from March to June 2018. Methodology: Patients who underwent screening CR mammography, followed by DR mammography a year later, were selected. Only disease-free individuals were included in the study. Evaluation of breast density was done subjectively, using the breast imaging reporting and data system (BI-RADS) by two independent experienced radiologists. Statistical analysis was performed using the Wilcox Signed Rank-sum test to compare both modalities. Fisher Exact method was used to compare the need for ultrasound imaging.

Results: A total of 295 patients were included in the study. The mean age of the patients was $52.76 \pm 0.64$ years. There was a significant difference in the change of breast density when comparing both modalities $(Z=-11.839, p<0.001)$. A statistically significant reduction in the need for further breast ultrasound was observed after DR mammography than with CR mammography $(p<0.001)$.

Conclusion: Use of DR mammography, especially in patients with dense breast parenchyma, is a better screening tool overall. It translates to better feasibility for the radiologist and is more economical for the patient. DR mammography decreases unnecessary imaging and leads to better visualisation, thus providing a more accurate categorisation of breast density.
\end{abstract}

Key Word: Computed radiography mammography, Breast density, Screening, Breast cancer, Digital mammography, Ultrasound.

How to cite this article: Saeed SA, Masroor I, Iqbal H, Sufian SN, Awais M. Variability of Breast Density Assessment and the Need for Additional Imaging: A Comparison between Computed Mammography and Digital Mammography. J Coll Physicians Surg Pak 2020; 30(11):1213-1216.

\section{INTRODUCTION}

Increased mammographic breast density is a known risk factor for breast cancer in addition to being a marker for decreased sensitivity for breast cancer detection. ${ }^{1}$ Increased mammographic density may also make it more difficult to diagnose breast cancer by mammography and can lead to an increase in risk for the development of breast cancer between subsequent screening examinations. ${ }^{2,3}$ However, computed radiography (CR) that uses phosphor plates and a separate reader, ${ }^{4}$ still remains to be the norm of practice for detecting breast cancer; and CR mammograms are at present the standard of care for screening programmes.

Correspondence to: Dr. Shaista Afzal Saeed, Department of Radiology, Aga Khan University Hospital, Karachi, Pakistan

E-mail: shaista.afzal@aku.edu

Received: December 30, 2019; Revised: January 04, 2020;

Accepted: February 22, 2020

DOI: https://doi.org/10.29271/jcpsp.2020.11.1213
Despite this, CR mammography has been criticised for showing a high proportion of false-positives and low sensitivity, particularly when mammograms show dense breast tissue. A recent multi-centre full field digital mammography (FFDM) revealed a sensitivity of $70 \%$ in contrast to $55 \%$ for CR mammograms with dense breasts, in those under 50 years of age $(p=0.0015) .{ }^{5}$ Mammography done with direct digital radiology (DR) system utilises an integrated X-ray system and detector. ${ }^{4}$ The images are transferred directly to the computer after the exposure and are reported to be better at detecting malignancy with dense breast and in high grade breast cancers. ${ }^{6}$ This has led to an increase in recognition of digital mammography by specialised breast radiologist in the last few years; and many have recommend converting from CR mammography to DR mammography. ${ }^{7}$ The breast imaging-reporting and data system (BI-RADS) density categorisation is done by visual or computer-aided determination of the percentage density (PD) of the breast and is frequently utilised to evaluate mammographic density. ${ }^{8}$ 
Table I: Relative mammographic density categorisation using computed mammography and digital mammography.

\begin{tabular}{|l|c|c|}
\hline Breast Parenchymal Pattern (Category) & Density using CR mammography (295) & Density using DR mammography (295) \\
\hline Fatty (1) & $9(3.1 \%)$ & $40(13.6 \%)$ \\
\hline Scattered fibro-glandular (2) & $114(38.6 \%)$ & $177(60 \%)$ \\
\hline Heterogeneously dense (3) & $149(50.5 \%)$ & $76(25.8 \%)$ \\
\hline Dense (4) & $23(7.8 \%)$ & $2(0.7 \%)$ \\
\hline
\end{tabular}

Table II: Need for ultrasound, CR vs. DR.

\begin{tabular}{|l|l|c|c|c|}
\hline \multicolumn{2}{|c|}{} & \multicolumn{2}{|c|}{ CR } & \multirow{2}{*}{ Total } \\
\cline { 2 - 5 } \multicolumn{2}{|c|}{ DR } & Yes & No & $78(26.44 \%)$ \\
\hline \multirow{2}{*}{ Total } & Yes & $78(26.44 \%)$ & $123(41.69 \%)$ & $217(73.56 \%)$ \\
\cline { 2 - 5 } & No & $94(31.86 \%)$ & $123(41.69 \%)$ & $295(100 \%)$ \\
\hline
\end{tabular}
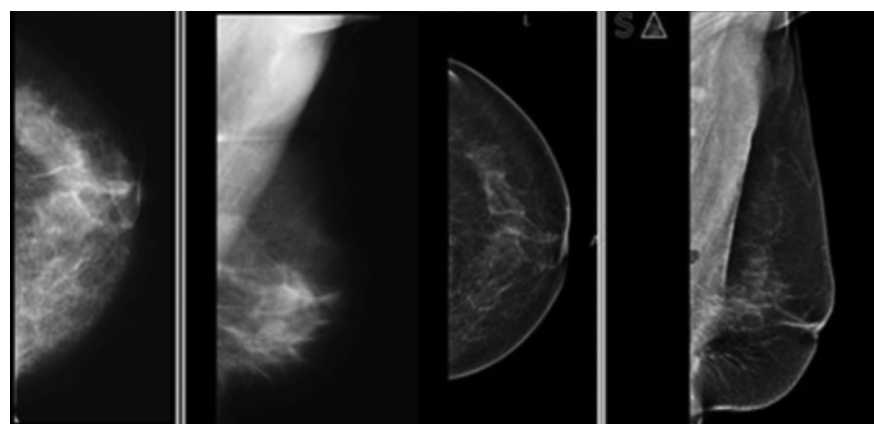

Figure 1: Left breast mammograms $(a, b)$ show hetrogenous dense breast parenchyma on CR mammography. Follow-up DR mammograms (c,d) a year later, show scattered fibro-glandular breast parencyme.
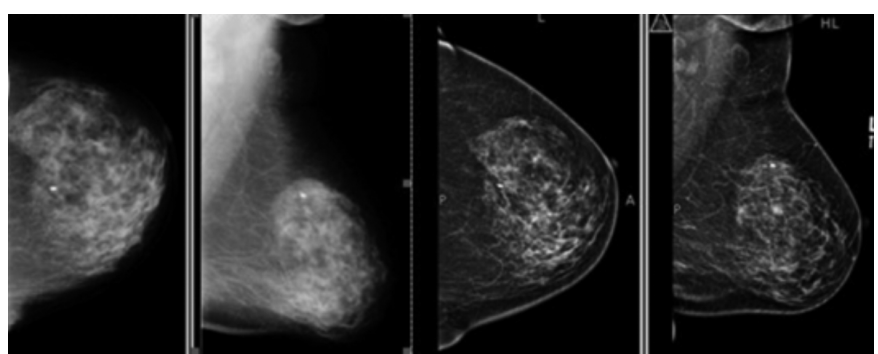

Figure 2: Left breast mammograms $(a, b)$ show hetrogenous dense breast parenchyma on CR mammography. Follow-up DR mammograms $(c, d)$ a year later, redemonstrate dense breast parencyme on DM.

To the knowledge of the authors, no such comparison has been performed between the use of DR mammography and CR mammography to gauge the variability of breast density in Pakistan. With this rationale in mind, the primary aim of this study was to assess the variability in breast density on screening mammograms comparing both these modalities. Secondary aim was to assess the need of further ultrasound examination in cases of dense breast, and time-and-cost analysis to ascertain the feasibility of implementation in the long run.

\section{METHODOLOGY}

The data was collected retrospectively by utilising a non-probability purposive consecutive sampling method. All patients undergoing DR mammography for routine screening, at the Radiology Department, The Aga Khan University Hospital, from March to June 2018 were selected. All these patients had underwent at least one screening mammogram a year earlier using CR mammography at the study centre. All patients undergoing diagnostic mammogram or undergoing screening mammograms for the first time were excluded. Patients whose screening mammogram revealed any abnormalities leading to further imaging or treatment were also excluded.

Subjective assessment of breast density, using a visual judgement method, was performed as the breast density quantitative measurement tool is not available in Pakistan. Breast density was categorised for each mammogram according to the BI-RADS breast density lexicon as fatty, scattered, fibro-glandular, heterogeneously dense and dense, in order to compare the differences between the two mammograms. Comparison of the recently performed DR mammograms was performed with the previously performed CR mammograms for each patient. Two independent radiologists, with greater than 10 years of experience in women imaging individually, interpreted the images. The results of density categorisations by the two radiologists were assessed; and any conflicts were resolved by another radiologist with greater than 15 years of experience in women imaging. This third radiologist was blinded to the categorisation assigned by the initial two radiologists. Statistical analysis was performed using IBM Statistical Package for the Social Sciences (SPSS) version $20 .^{9}$

Descriptive variables were expressed as relative frequencies and percentages. Quantitative variables were expressed as mean \pm standard deviation and median (IQR). Normality of data was checked through the Shapiro-Wilk test. Wilcoxon Rank test was used to compare the categories of breast density assigned using CR mammography versus DR mammography. Fisher Exact test was used to compare the need for ultrasound in the two groups. A p-value of $<0.05$ was considered statistically significant.

\section{RESULTS}

A total of 512 patients underwent digital mammograms between March to June 2018. Out of these, 295 (57.6\%) patients had a screening mammogram done by the computed radiography system from the Department a year ago. All 
these patients were included in the study as they had disease-free image results. The mean age of the patients was $52.76 \pm 11.11$ years. The difference in breast density parenchymal pattern by CR mammography and DR mammography is shown in Table $\mathrm{I}$.

The median (and interquartile range) for breast parenchymal density for CR and DR were 3 (2-3) and 2 (2-3), respectively. Wilcoxon Rank test showed a statistically significant difference in the breast parenchymal density categorisation in patients who underwent DR mammography when compared to CR mammography $(Z=-11.839 ; p<0.001)$. DR mammography resulted in lowering the breast density category in 143 $(48.5 \%)$ patients. There were no instances of higher breast density categorisation using DR mammography.

The median (and interquartile range) for BI-RADS categorisation for CR and DR were 0 (0-2) and 2 (1-2), respectively. Wilcoxon Rank test showed a statistically significant difference in the BI-RADS categorisation in patients who underwent DR mammography when compared to CR mammography ( $Z=$ -9.022; $p<0.001$ ). DR mammography resulted in increasing the BI-RADS category in $118(40 \%)$ patients, and decreasing the BI-RADS category in $11(3.73 \%)$ patients as compared to CR mammography.

For categories of heterogeneously dense and dense parenchyma, routine ultrasound examination was performed to detect underlying occult lesions, as higher breast densities reduce sensitivity of mammogram. Fisher Exact test showed that there was a significant reduction $(p<0.001)$ in the need for ultrasound after DR mammography in 78 (26.44\%) patients as compared to 172 (58.31\%) patients with CR mammography (Table II).

\section{DISCUSSION}

Breast density refers to the quantity of fibro-glandular tissue present in the breast, relative to the amount of fat. Breast density evaluation is a crucial part of any screening mammography report, as it assists the clinician in assessing the sensitivity of a mammogram to detect the relative risk of breast cancer. Frequently used techniques of mammographic density evaluation vary in subjective visual estimation to quantitative calculation of area and volume density percentages, with the help of complex mathematical algorithms. The fifth edition of American College of Radiology has now changed their BI-RADS lexicon categorisation system by removing the technique that utilised approximation of breast density percentage quartiles. ${ }^{10}$ Instead of using an overall approximation of the proportion of glandular and stromal breast, the present BI-RADS revision has a more subjective approach. By adopting a four-category-based evaluation system for breast composition, the potential for detection of cancerous lesions in denser areas of the breast is greater. As most experts agree that, a heterogeneously dense breast and breast with extremely dense fibro-glandular tissue (more than $50 \%$ dense, according to BI-RADS fourth edition) are cate- gorised as dense; wheraeas, breasts with scattered fibro-glandular tissue and fatty parenchymal pattern are grouped as non-dense. While dense tissue is linked with an increased risk of breast cancer, the degree of this risk is usually not known, because it depends on other underlying factors such as age, endogenous hormones exposure etc. ${ }^{11}$

Approximately $50 \%$ of the screened population tend to have dense breast tissue. Due to the reduced sensitivity of screening mammography in dense breast parenchyma, ${ }^{12}$ additional imaging is usually advised either in the form of ultrasound, MRI or both. In females with known cancer, evaluation with ultrasound and $M R$ in addition to mammography becomes part of the triple assessment. ${ }^{13}$ In foresight, this adds on to the workload of the radiologist and increases medical cost for the patient or the healthcare system involved. In the present study, 295 women who underwent both CR mammography and DR mammography after an interval of one year, showed statistically significant reduction in density categorisation by the use of DR mammography (Figure 1), thus proving digital mammography to have better sensitivity than conventional computed aided mammograms. Similar results were achieved by Fischmann et al. in which they compared parenchymal breast densities on film screen mammography with full field digital mammography. ${ }^{14}$ All three radiologists involved in their study classified the breast parenchyma to be less dense on digital mammograms. The improved performance with DR is most likely related to image acquisition, display and processing of digital data. With DR, the image contrast can be manipulated, thus allowing optimisation and increasing contrast in dense areas of the breast. ${ }^{15}$

There was a significant reduction in the need for ultrasound imaging, using DR mammography, owing to the fact that fewer mammograms were classified as being dense (78 patients) with DR mammography as compared to 172 patients with CR mammography; $p=.001$, Figure 2. A total of 94 patients were spared from undergoing additional ultrasound examination.

On average, the procedure time for bilateral breast ultrasound is around 20 minutes at this institute; this translated to a total of approximately 31 work hours saved for the radiologist, during the study period. The cost of bilateral breast ultrasound at the study centre is Rs.7560 (USD 48.32), thus a potential saving of Rs.710640 (USD 4532) was observed by all the patients, included in the study. Since Pakistani population mainly adopts an out-of-pocket healthcare system, a substantial advantage of DR mammography was noted.

In the absence of population-based screening for breast cancer in Pakistan, digital mammography is thus far superior, when assessing dense breast tissue, to computed mammography; however, this preference is only well established in females less than 50 years of age. ${ }^{16}$ Due to a higher propensity of breast cancer in Pakistani/Asian population, the use of digital mammography by radiologists is advocated as a routine practice. 
There are a few limitations within this study. Firstly, the assessment of breast density was purely subjective and, hence, could not account for inter-observer variations. Blinding of the radiologist for the CR mammography and subsequent DR mammography of the same participant was not possible; this in itself could have created a bias in assigning a breast density category.

These factors may have affected the outcome of this study. Despite this, the plausible differences in breast density, when comparing both modalities, are still apparent.

\section{CONCLUSION}

As DR mammography becomes the mainstay screening tool around the world for breast cancer detection, a clear advantage of using DR mammography over CR mammography was also observed in the present study. The time and financial cost saved by this modality, due to better visualisation, is beneficial to both the patient and healthcare providers as a whole.

\section{ETHICAL APPROVAL:}

Study was approved as exemption by Ethics Review Committee, The Aga Khan University (2019-0125-2196).

\section{PATIENT'S CONSENT:}

Since this was a retrospective review of imaging and the data was anonymised, hence informed consent was not required.

\section{CONFLICT OF INTEREST:}

Authors declared no conflict of interest.

\section{AUTHORS' CONTRIBUTION:}

SA, IM: Contributions to the conception and design of the work, data acquisition, analysis and interpretation of data.

HI: Contributions to the conception and design of the work, data acquisition.

$\mathrm{SN}$ : Contribution to the cases, image interpretation and final approval.

MA: Data analysis, drafting the work, revising it critically for important intellectual contents.

\section{REFERENCES}

1. Harvey JA, Bovbjerg VE. Quantitative assessment of mammographic breast density: Relationship with breast cancer risk. Radiology 2004; 230(1):29-41. doi: 10.1148/radiol.2301020870.

2. Sala E, Warren R, McCann J, Duffy S, Day N, Luben R. Mammographic parenchymal patterns and mode of detection: Implications for the breast screening programme. J Med Screen 1998; 5(4):207-12. doi: 10.1136/ jms.5.4.207.

3. Van Gils CH, Otten JD, Verbeek AL, Hendriks JH. Mammographic breast density and risk of breast cancer: Masking bias or causality. Eur J Epidemiol 1998; 14(4):315-320. doi: 10.1023/a:1007423824675.

4. Bosmans H, De Hauwere A, Lemmens K, Zanca F, Thierens $\mathrm{H}$, Van Ongeval $\mathrm{C}$, et al. Technical and clinical breast cancer screening performance indicators for computed radiography versus direct digital radiography. Euro Radiol 2013; 23(10):2891-8. doi: 10.1007/ s00330-013-2876-0.

5. Pisano ED, Hendrick RE, Yaffe MJ, Baum JK, Acharyya S, Cormack JB, et al. Diagnostic accuracy of digital versus film mammography: exploratory analysis of selected population subgroups in DMIST. Radiology 2008; 246(2):376-83. doi: 10.1148/radiol.2461070200.

6. Séradour B, Heid P, Estève J. Comparison of direct digital mammo-graphy, computed radiography, and film-screen in the French national breast cancer screening program. Am J Roentgenol 2014; 202(1):229-36. doi: 10.2214/AJR. 12.10419.

7. Yaffe MJ. Mammographic density. Measurement of mam-mographic density. Breast Cancer Res 2008; 10(3):209.

8. Pisano ED. Digital compared with screen-film mammo-graphy: Measures of diagnostic accuracy among women screened in the ontario breast screening program-evidence that direct radiography is superior to computed radiography for cancer detection. Radiology 2016; 278(2):311-2. doi: 10.1148/radiol.2015152344.

9. Available from:http: //www.ibm.com/analytics/spss- statisticS-software. Last visited 24th November 2019

10. D’Orsi CJ, Mendelson EB, Ikeda DM. Breast Imaging Reporting and Data System, 5th. Reston, VA, USA: American College of Radiology 2013.

11. Winkler NS, Raza S, Mackesy M, Birdwell RL. Breast density: Clinical implications and assessment methods. Radiographics 2015; 35(2):316-24. doi: 10.1148/rg. 352140134.

12. Lee $\mathrm{Cl}$, Bassett LW, Lehman CD. Breast density legislation and opportunities for patient-centered outcomes research. Radiology 2012; 264(3):632-6. doi: 10.1148/ radiol.12120184.

13. Fatima S, Waheed S, Khan MI. Diagnostic accuracy of Mr Mammography in diagnosing malignant breast lesions taking histopathology as gold standard. J Coll Phys Surg Pak 2019; 29(1):16-8. doi: 10.29271/jcpsp.2019.01.16.

14. Fischmann A, Siegmann KC, Wersebe A, Claussen CD, Muller-Schimpfle M. Comparison of full-field digital mammography and film-screen mammography: Image quality and lesion detection. Brit J Radiol 2005; 78(928): 312-5. doi: $10.1259 / \mathrm{bjr} / 33317317$.

15. Pisano ED, Gatsonis C, Hendrick E, Yaffe M, Baum JK, Acharyya $S$, et al. Diagnostic performance of digital versus film mammography for breast-cancer screening. N Eng J Med 2005; 353(17):1773-83. doi: 10.1056/NEJMoa 052911.

16. Souza FH, Wendland EM, Rosa MI, Polanczyk CA. Is full-field digital mammography more accurate than screen-film mammography in overall population screening? A systematic review and meta-analysis. Breast 2013; 22(3):217-24. doi: 10.1016/j.breast.2013.02.013. 